\title{
Translation and Psychometric Evaluation of the Chinese Adjustment Disorder - New Module 20
}

\author{
Louisa Lorenz ${ }^{1,2}$; Grace W.K. Ho ${ }^{3}$; Athena C.Y. Chan ${ }^{3}$; Daniel T. Bressington ${ }^{3}$; \\ Wai Tong Chien ${ }^{4}$; Mark Shevlin5; Philip Hyland ${ }^{6}$; Andreas Maercker ${ }^{7}$; Thanos Karatzias ${ }^{8,9}$ \\ ${ }^{1}$ Department of Psychology, University of Zurich, Zurich, Switzerland \\ ${ }^{2}$ Klinik im Hasel, Stationaere Therapie, Gontenschwil, Switzerland \\ ${ }^{3}$ School of Nursing, The Hong Kong Polytechnic University, Hong Kong SAR \\ ${ }^{4}$ The Nethersole School of Nursing, The Chinese University of Hong Kong, Hong Kong SAR \\ ${ }^{5}$ School of Psychology, Ulster University, Derry, Northern Ireland \\ ${ }^{6}$ Department of Psychology, Maynooth University, Kildare, Ireland \\ ${ }^{7}$ Department of Psychology, University of Zurich, Zurich, Switzerland \\ ${ }^{8}$ Edinburgh Napier University, School of Health \& Social Care, United Kingdom \\ ${ }^{9}$ NHS Lothian, Rivers Centre for Traumatic Stress, United Kingdom
}

\section{Corresponding Author:}

Grace W.K. Ho, PhD, RN

PQ426, The Hong Kong Polytechnic University, Hung Hom, Hong Kong

+85234003918| grace.wk.ho@polyu.edu.hk 


\title{
Translation and Psychometric Evaluation of the Chinese Adjustment Disorder - New Module 20
}

\begin{abstract}
The Adjustment Disorder - New Module 20 (ADNM-20) was developed for the assessment of adjustment disorder symptoms. The aim of the present study was to translate and conduct psychometric testing of the Chinese version of the ADNM-20. The English version was translated into traditional Chinese in an iterative process with an expert panel of mental health professionals. A total of 433 university students in Hong Kong between ages 18-24 completed the Chinese ADNM-20 and the Hospital Anxiety and Depression Scale via an online survey; 32 were retested after two weeks. The content validity indices were excellent for most of the items. Confirmatory factor analysis supported a unidimensional first-order structure with excellent composite reliability. The test-retest reliability of the Chinese version was below satisfactory for the individual items; the intra-class correlation for the overall scale was moderate. The Chinese version of the ADNM-20 also indicated a moderate positive correlation with anxiety and depression symptoms. Initial results support the content, factorial, and discriminant validity of the translated scale. More research is needed to assess its reliability in this cultural context. The ADNM-20 can be quickly employed for the assessment of adjustment disorder as proposed for ICD-11.
\end{abstract}

Keywords: Adjustment Disorder; ICD-11; Clinical Assessment; Validation; Confirmatory Factor Analysis (CFA) 


\section{Background}

Adjustment disorder (AjD) is defined as the development of emotional and behavioural symptoms in response to a critical life event (World Health Organization, 1992). A major issue with the current definition of $\mathrm{AjD}$ is that the International Classification of Diseases (ICD), $10^{\text {th }}$ revision (World Health Organization, 1992), and the Diagnostic and Statistical Manual, $5^{\text {th }}$ revision (DSM-5; American Psychiatric Association, 2013) do not define clear symptom criteria for the diagnosis of AjD (Strain \& Diefenbacher, 2008). Instead, the diagnosis is made if symptoms of depressive, anxiety, neurotic, stress-related, somatoform, and/or conduct disorders are present but do not meet the full threshold of an individual diagnosis (World Health Organization, 1992). This loose symptom definition led to inadequate assessment methods for AjD (Casey, Dowrick, \& Wilkinson, 2001). Until recently, there were no instruments specifically designed for the assessment of AjD as a sub-threshold diagnosis (Casey et al., 2001).

During the revision of the ICD and DSM, a new concept of AjD that included a positive symptom formulation was proposed (A. Maercker, Einsle, \& Köllner, 2007). In the course of this revision, a new self-report questionnaire was developed: the Adjustment Disorder - New Module (ADNM; A. Maercker et al., 2007). The ADNM contains two parts: the first part consists of a list of 19 possible critical life events, on which individuals indicate their exposure over the past year; the second part is a list of possible $\mathrm{AjD}$ symptoms in response to the most stressful event. In its original version, the symptom list contained 29 items (ADNM-29; Einsle, Köllner, Dannemann, \& Maercker, 2010) but with further revision of the scale, the questionnaire was reduced to 20 items (ADNM-20; Glaesmer, Romppel, Brähler, Hinz, \& Maercker, 2015). Both versions measure six symptoms areas: (1) preoccupation with the stressor (PRE), (2) failure to adapt (FTA), (3) 
avoidance, (4) depression, (5) anxiety, and (6) impulsivity. The ADNM-20 also includes one item that measures functional impairment.

A 6-factor structure was supported in prior psychometric testing of the ADNM-29 (Einsle et al., 2010) and ADNM-20 (Glaesmer et al., 2015). However, due to high first-order factor correlations, a unidimensional structure was proposed for the ADNM-20 (Glaesmer et al., 2015; Lorenz, Hyland, Perkonigg, \& Maercker, 2018). As ICD-11 only focuses on the core symptoms (Andreas Maercker et al., 2013), Zelviene, Kazlauskas, Eimontas, and Maercker (2017) proposed a 2-core factor model for the items reflecting PRE and FTA. During the initial validations, the ADNM-20 showed sufficiently good reliabilities, with Cronbach's alphas ranging from $\alpha=.81-.94$ (L. Lorenz, R. Bachem, \& A. Maercker, 2016). Furthermore, Einsle et al. (2010) reported the testretest reliability of the ADNM-29 of $\mathrm{r}_{\mathrm{tt}}=.61-.84$ over a 6-week interval. Previous studies on the ADNM-29 also found moderate associations with the Hospital Anxiety and Depression Scale (Einsle et al., 2010), the Montgomery-Asberg Depression Scale (Bachem, Perkonigg, Stein, \& Maercker, 2017), and the Anxiety Rating Scale (Bachem et al., 2017) as indicators of discriminant validity.

Although much research has been devoted to understanding and assessing $\mathrm{AjD}$ in the past two decades, most were conducted in Western settings and little attention has been given to examining AjD in Asian populations. In fact, among the relatively small number of investigations on $\mathrm{AjD}$ in Asian samples, most have primarily focused on Asian Americans rather than native East Asian groups (Chun \& Hsu, 2012). Nonetheless, it is clear that culture can be a strong determinant for how individuals adjust and respond to stressors. For example, prior studies have reported that the cultural stigma attached to certain stressors (e.g. divorce and domestic violence) in Chinese populations may lead to more complicated responses to these stressors, such as suppression of true 
feelings and fear of social exclusion, which may further lead to denial of maladaptive responses or preclude overall positive adjustment in the face of those specific stressors (Anderson \& Mayes, 2010; Chan, Lam, \& Shae, 2011; Chun \& Hsu, 2012). Importantly, most studies that examined maladjustment in Chinese populations were based on measures of other nonspecific psychological distress (e.g. general depressive or anxiety symptoms, or psychosocial dysfunction) (Gau, Chong, Chen, \& Cheng, 2005; Shek, Chan, \& Wong, 2012; Tu, Hsu, Chi, Lin, \& Yen, 2014), and no known instrument specifically designed to assess $\mathrm{AjD}$ has been validated for use in Chinese samples.

The aim of the present study was to develop a Chinese version of the ADNM-20 and to test its psychometric properties in a convenience sample of Chinese university students. We evaluated four characteristics of the questionnaire: (1) the content validity of the Chinese translation, (2) the factor structure of the items, (3) the composite and the test-retest reliability, and (4) the association of the ADNM-20 with depression and anxiety as discriminant validity of the questionnaire.

\section{Materials and Methods}

\section{Phase 1: Chinese Translation and Content Validation}

Translation of the ADNM-20 was conducted similar to the steps described by Beaton, Bombardier, Guillemin, and Ferraz (Beaton, Bombardier, Guillemin, \& Ferraz, 2000). The ADNM-20 was translated from English to traditional Chinese by a bilingual technical writer, and back-translated by a bilingual study team member. Three other study team members who are experienced in mental health research independently reviewed the initial forward and backward translations. Two additional iterations of the translations were generated before a consensus was reached for the initial draft. 
The initial translation of the AjD symptom items was assessed for content validity in terms of their relevance to the theme and their appropriateness to the Chinese culture (Polit \& Beck, 2006; Polit, Beck, \& Owen, 2007). An expert panel of two mental health researchers, two clinical psychologists, and two social workers who were not part of the study team rated the relevance and appropriateness of each translated question on a 4-point Likert scale, ranging from 4 ('highly relevant/appropriate'), 3 ('quite relevant/appropriate'), 2 ('somewhat relevant/appropriate'), to 1 ('not relevant/appropriate'). After content validation, the Chinese ADNM-20, including items on current life stressors and AjD symptoms, was pilot tested with eight young adults recruited from a university setting, and all eight participants provided positive comments on the clarity, understandability, and ease of answering the questions. The final translated version of the scale was deployed for a larger psychometric evaluation.

\section{Phase 2: Psychometric Evaluation}

\section{Participants and settings}

Young adults aged 18 to 24 , who were enrolled in an undergraduate degree program in Hong Kong, and who were able to read traditional Chinese were eligible to participate in this study. They were recruited via convenience sampling from two major universities and their affiliated community colleges in Hong Kong. Flyer with study information were posted on message boards and distributed around college campuses. Participants entered the study website to answer the survey questions anonymously. They were asked to supply their contact information if they agreed to be contacted again for a study follow-up. Among $N=433$ participants who completed the Chinese version of the ADNM-20 online, $n=32$ completed the retest. This retest sample size is sufficient to detect intra-class correlation of 0.50 or above at $90 \%$ power and alpha of 0.05 for 2 observations per subject (Bujang \& Baharum, 2017). For the full sample, the mean age of the 
participants was $M=20.16(S D=1.67)$. Over half of the participants were female (58.9\%) and approximately half were enrolled in an associate degree program (50.3\%). For the retest, the mean age was $M=20.81(S D=1.70)$; twenty-four were female ( $75.8 \%)$ and thirteen were associate degree students $(39.4 \%)$.

This study was approved by the ethics committee of the Hong Kong Polytechnic University. MySurvey v1.1 (The Hong Kong Polytechnic University, 2016) was used to collect data online. The study information included that informed consent was implied by survey completion, and data was immediately stored in a secure backend database. To complete the ADNM-20, participants indicated their exposure to a list of 19 life stressors and were asked to answer the AjD symptom items based on the most straining event(s). Participants also completed measures of their depression and anxiety. At survey completion, participants may provide their contact information if they agree to participate in a follow-up survey and/or for a chance to be entered into a lucky draw to win an electronic tablet. At approximately two weeks after initial survey, participants who agreed to be contacted again were approached to complete the Chinese ADNM-20 again.

\section{Measures}

The Adjustment Disorder - New Module 20 (Einsle et al., 2010) consists of two parts. First, participants were asked to indicate exposure to stressful critical life events in the past year that have burdened them in the past six months from a list of 19 potential stressors. Second, participants responded to $19 \mathrm{AjD}$ symptom indicators plus 1 item that reflects functional impairment. The response format is a 4-point Likert scale ranging from 1 ('never') to 4 ('often'); sum scores are used for either all items or on subscale level. 
The Hospital Anxiety and Depression Scale (HADS; Zigmond \& Snaith, 1983) was used to measure anxiety and depression for the assessment of convergent and discriminant validity. The HADS consists of 14 items that are answered on a 4-point Likert scale ranging from 0 to 3 with response options varying by item. The depression and anxiety subscales include 7 items each, with higher sum scores reflecting higher levels of symptomatology. The Chinese version of the HADS was previously validated in Hong Kong samples with regard to factor structure, internal consistency, retest reliability, and convergent/discriminant validity (Li et al., 2016; Wang, Chair, Thompson, \& Twinn, 2009).

\section{Statistical analysis}

Content validity indices were calculated for relevance and appropriateness both by item and for the overall questionnaire. Item-level analysis was conducted using item content validity index (I-CVI), which is the proportion of experts who rated the item with a score of 3 or 4 (out of 4) (Polit \& Beck, 2006). A modified kappa statistic $\left(k^{*}\right)$ was computed to correct for the chance agreement among experts that might artificially inflate the I-CVI ratings (Polit et al., 2007). Content validity index for the overall scale (S-CVI) was computed using two methods: (1) universal agreement (S-CVIUA), which is the proportion of items that received a score of 3 or 4 by all experts, and (2) an average I-CVI of all scale items (S-CVI $\left.{ }_{\text {Ave }}\right)$ (Polit \& Beck, 2006). For a panel of six raters, I-CVI, S-CVI $I_{\mathrm{UA}}$, and $\mathrm{S}-\mathrm{CVI}_{\mathrm{Ave}}$ are considered good when coefficient exceeds 0.78, 0.80, and 0.90, respectively (Lynn, 1986; Polit \& Beck, 2006); $\mathrm{k}^{*}>0.74$ is considered excellent (Polit et al., 2007).

The factor structure of the ADNM-20 symptom items was investigated using confirmatory factor analysis. In total, four models that were recently proposed in the literature were tested in the

present study. Three models tested the structure of all 19 symptom indicators, while one model 
only used the 8 items that are designed to measure PRE, FTA, and functional impairment. Following the example of Lorenz et al. (2018), a bifactor model with one general factor explaining covariation across all items (Reise, 2012) would have been tested, if the best fitting first-order solution was multidimensional. The first model was a single factor solution in which all 19 symptom indicators load on an AjD factor (1-factor model) as proposed by Glaesmer et al. (2015). The 5-factor model included five correlated general factors reflecting PRE (4 items), FTA (3 items), avoidance (4 items), affective reaction (5 items), and impulsivity (3 items) (Lorenz et al., 2018). The 6-factor model represented the basic six-factor solution as proposed by Einsle et al. (2010) and reflected PRE, FTA, avoidance, depression (3 items), anxiety (2 items), and impulsivity. The model testing the reduced item range, the 2-core-factor model (Zelviene et al., 2017), contained two factors reflecting PRE and FTA with 4 items each (the item measuring functional impairment loaded on FTA). All models were tested using Mplus, Version 8.1 (L. K. Muthén \& Muthén, 2017) using the robust weighted least squares, mean- and variance-adjusted (WLSMV) estimator (Karl G. Jöreskog, 1994; B. O. Muthén, 1997). We followed standard recommendations for assessing model fit (Jackson, Gillaspy Jr, \& Purc-Stephenson, 2009): Acceptable model fit was defined as a non-significant chi-square, a comparative fit index (CFI) and Tucker-Lewis Index (TLI) greater than .95. For the Root-Mean Square Error of Approximation (RMSEA) values less than .05 represent 'close' fit and up to .08 indicated 'reasonable' errors of approximation (Karl G Jöreskog \& Sörbom, 1993). The same cut-off values were used for the Standardised Root Mean Square Residual (SRMR) (Karl G Jöreskog \& Sörbom, 1981). The fit of the CFA models was compared using the DIFFTEST procedure in Mplus. The adequacy of models was also assessed by inspection and interpretation of the parameter estimates: improper solutions 
or out-of-bounds estimates were considered to be indicative of misspecified models (Chen, Bollen, Paxton, Curran, \& Kirby, 2001).

We computed the composite reliability for the resulting models using the Composite Reliability Calculator (Colwell, 2016). Test-rest reliability over 2 weeks was assessed by percentage agreement (PA), with $\mathrm{PA} \geq 70 \%$ considered satisfactory (Kazdin, 1977). Systematic disagreement between paired ordinal item scores was further analysed using the rank-invariant method developed by Svensson (Svensson, 2012). This method estimates the relative position (RP), i.e. the degree of which the distribution of scores from one assessment is systematically shifted to a higher or lower score in another assessment, and relative concentration (RC), i.e. the degree of which the distribution of scores from one assessment is more or less concentrated towards the central scale categories (Svensson, 2012). Possible values of RP and RC range from 1 to 1; a higher absolute value of RP implies a greater systematic difference between assessments, and a higher absolute value of RC implies the score of an assessment is systematically more concentrated than another assessment. The individual variability was assessed by relative rank variance $(\mathrm{RV})$, which is defined by the sum squares of rank differences when the ranks are tied to the pairs of observations (Svensson, 2012); RV $<0.1$ is generally regarded as negligible. We further examined test-retest reliability using two-way mixed effects model intra-class correlation coefficients (ICC; DeVon et al., 2007; Koo \& Li, 2016). These analyses were conducted using STATA IC14.

Lastly, we assessed concurrent and discriminant validity for the best fitting solution for all 19 symptom indicators plus only for the core symptoms. We computed the unique and partial correlations between each latent variable and the two manifest criterion variables, i.e. depression and anxiety. 


\section{Results}

\section{Life Events and AjD symptomatology}

A total of 433 participants completed the Chinese version of the ADNM-20 and reported an average of $3.2(S D=1.8)$ critical life events in the past year. The most commonly reported life events were pressure to meet deadlines $(n=360,83.1 \%)$, adjustment to tertiary education ( $n=191$, $44.1 \%)$, financial problems $(n=167,38.6 \%)$, too much work $(n=166,38.3 \%)$, family conflicts $(n=137,31.6 \%)$, and work-life conflicts $(n=124,28.6 \%)$. Participants' mean scores were $M=41.7$ $(S D=13.2$, Range $=20-78)$ on the ADNM-20, $M=6.9(S D=3.4)$ on depression, and $M=9.2(S D=3.7)$ on anxiety. Nearly one-third of the participants $(n=130,30.0 \%)$ were at high risk for AjD based on the suggested cut-off score of 47.5 or above (L. Lorenz, R. C. Bachem, \& A. Maercker, 2016), Females reported a significantly higher score on the ADNM-20 $(M=43.0, S D=13.6)$ compared to males $(M=39.9, S D=12.5 ; t(431)=-2.461, p<.01, d=0.24)$; a higher proportion of female participants $(n=87,34.1 \%)$ were at high risk for $\mathrm{AjD}$ compared with males $(n=43,24.2 \%)$.

\section{Content validity}

Table 1 summarizes the findings regarding content validity. Overall, 18 of the 20 translated items $(90 \%)$ of the ADNM-20 received excellent ratings on relevance and appropriateness. The remaining two items that measure preoccupation received fair ratings for appropriateness. Scalelevel content validity was high, with $\mathrm{S}-\mathrm{CVI}_{\mathrm{UA}}=0.85$ and $\mathrm{S}-\mathrm{CVI}_{\mathrm{Ave}}=0.99$ for relevance, and $\mathrm{CVI}_{\mathrm{UA}}=0.80$ and $\mathrm{S}-\mathrm{CVI}_{\mathrm{Ave}}=0.95$ for appropriateness. Because two items measuring PRE received low appropriateness ratings, the study team members examined the translations in detail and found that raters were largely concerned with the meaning of 'I have to think about the stressful situation...' and noted that 'have to' should be omitted. However, the team decided to retain the original translation for two reasons. First, the term "have to" reflects the involuntary and 
uncontrollable nature of preoccupation and, second, the term was retained to ensure translational equivalence between the English and Chinese versions.

\section{Factorial validity}

The results of the confirmatory factor analysis are displayed in Table 2 . The model that included all symptoms appeared to fit the data reasonably well. Although the model chi-square statistic was significant, this should not lead to rejection as the power of the chi-square test is positively related to sample size (Tanaka, 1987). Also, the RMSEA was too high for this 1-factor model. Based on inspection of modification indices and model residuals, it was evident that the model was not adequately accounting for the covariation between items 9 ('Since the stressful situation, I lose my temper much quicker than I used to, even over small things') and 12 ('I have noticed that I am becoming more irritable due to the stressful situation'). These were both similar in that they measured anger based emotional regulation difficulties. To account for this, a correlated error was added to each model and the models were re-estimated. All these models demonstrated acceptable model fit, but the factor correlations for the multi-dimensional models were all very high. Of the 10 factor correlations in the 5-factor model, 2 were greater than 1 (outof-bounds estimates), 4 were $>.90$, and the remaining correlations ranged from to .809 to .854 . Of the 15 factor correlations in the 6-factor model, 5 were greater than 1 (out-of-bounds estimates), 6 were $>.90$, and the remaining correlations ranged from to .809 to .870 . The out-of-bounds estimates indicted that the multi-dimensional models were misspecified, and the magnitude of the correlations were indicative of a 1-factor model. The fit of the 1-factor model with one correlated error was acceptable and the DIFFTEST showed that it was significantly better than the 1 -factor model without the correlated error $(\chi 2(1)=82.902, \mathrm{p}<.001)$. On the basis of these considerations 
the 1 -factor model was considered the best model ${ }^{1}$. An exploratory factor analysis was also conducted using WLSMV estimation and the eigenvalues for the sample correlation matrix indicated only 2 factors with an eigenvalue $>1$ (factor $1=10.470$, factor $2=1.258$ ) and the loadings for all items on first factor were all higher than the loadings on the second factor based on the Geomin rotated solution. This provided more evidence for the unidimensional structure of the scale.

With regard to the core symptoms, the 2-core-factor model exhibited acceptable model fit across the majority of indices. All items showed positive and statistical significant factor loadings on their respective factors. The PRE and FTA factor correlated at $r=.92$.

\section{Reliability}

The composite reliability of the 1 -factor model was excellent with $\mathrm{CR}=.96$. For the 2-corefactor model, composite reliability was high with $\mathrm{CR}=.88$ for $\mathrm{PRE}$ and $\mathrm{CR}=.75$ for FTA. Table 3 summarizes the findings regarding test-retest reliability. The time between assessments ranged from 14 to 31 days $(M=22.2, S D=6.2)$. Overall, PA was below satisfactory $(<70 \%)$ for all items. Of the 20 items, six had a small but significant systematic disagreement between responses at initial test and retest. The ICC for the overall scale was moderate (ICC $=0.74[0.54 ; 0.87])$.

\section{Discriminant validity}

The AjD factor in the 1-factor model correlated positively and moderately with depression $(r=.54)$ and anxiety $(r=.68)$. For the 2 -core-factor model, PRE showed a weak to moderate positive association with depression $(r=.49)$ and a moderate positive association with anxiety $(r=.66)$. FTA was moderately positive associated with both depression $(r=.61)$ and anxiety $(r=.67)$. 


\section{Discussion}

The purpose of this study was to provide a psychometric evaluation of the first Chinese version of the Adjustment Disorder - New Module 20. Overall, results provide preliminary indications that the translated ADNM-20 is valid, relevant, and acceptable among young adults in the Chinese culture. The Chinese translation was created in an iterative process and the content validity of the items was overall satisfactory. Two items reflecting PRE were criticized because of a strict wording with more rigid implications ('have to'), however, they were retained in the questionnaire. In the further analysis, item 2 evidenced systematic disagreement between test and retest. As PRE is one of the core symptoms proposed for the diagnostic criteria in ICD-11 (Andreas Maercker et al., 2013), it might be beneficial to further work on the specific item formulation to make the questions more acceptable in the Chinese culture.

Confirmatory factor analysis supported a unidimensional first-order model for all 19 symptom indicators. Since the six-factor model was proposed in the initial validation of the ADNM (Einsle et al., 2010), several studies have provided evidence for a unidimensional conceptualization of AjD (Einsle et al., 2010; Lorenz et al., 2018). Our results suggest that the symptoms of maladjustment to life stressors may also be reflected as a single entity in the Chinese culture. However, ICD-11 defines PRE and FTA as separate entities (World Health Organisation, 2018) and, similar to an earlier study (Zelviene et al., 2017), the 2-core factor model showed good fit with the data and performed well with exogenous variables in the present analysis. Thus, further work is required to decrease the number of items that describe the core symptoms of the condition. The specific distinction between PRE and FTA symptoms should be investigated in future studies.

A related issue concerns the contribution of item 20, which is supposed to measure impairment in functioning. In previous validations, this item loaded on the FTA subscale because interference 
of the symptoms with everyday functioning is part of the FTA definition (Andreas Maercker et al., 2013). However, impairment in social, occupational, and other important areas of functioning is considered as an additional diagnostic requirement for AjD (World Health Organisation, 2018), which provides a sound rationale to detach item 20 from the FTA subscale (Lorenz et al., 2018). Therefore, future revisions of the questionnaire should address the issue of measurement conflation between FTA symptoms that interfere with everyday function and impairment in functioning as diagnostic criterion.

Recent studies have also investigated the shortening of the ADNM-20 and two brief measures, the ADNM-8 and the ADNM-4, were developed (Ben-Ezra, Mahat-Shamir, Lorenz, Lavenda, \& Maercker, 2018; Lavenda et al., 2019; Zelviene et al., 2017). The reasoning for the development of an abbreviated form was to focus on the proposed ICD-11 core symptoms as well as the evidence for a unidimensional structure in previous studies (Einsle et al., 2010; Lorenz et al., 2018). Both abbreviations, the ADNM-8 and the ADNM-4, evidenced satisfactory construct validity, convergent and discriminant validity, and good accordance with the long version (BenEzra et al., 2018; Lavenda et al., 2019; Zelviene et al., 2017). This study used the longer version with 20 items to account for cultural differences in the phenomenology of adjustment difficulties. Since there was again evidence for unidimensionality of the measure in the current study, future research should focus on the development and testing of an abbreviated ADNM measure for the Chinese culture.

The test-retest reliability of the Chinese version of the ADNM-20 in this study was not acceptable. There was a low agreement between the test and retest scores and statistically significant disagreement in half of the items. One reason for the weak results could lie in the design of the study. AjD is a condition, in which symptoms are fluctuating and diminishing over time. 
This could account for the low agreement between test and retest conducted approximately two weeks apart. Moreover, the first assessment was during the academic year while the second assessment was during the summer break which could have accounted for lower stress levels at retest. Hence, present results should be interpreted with caution and future validation should account for these issues to provide a more robust picture of retest reliability. Lastly, the analysis regarding discriminant validity of $\mathrm{AjD}$ revealed that $\mathrm{AjD}$ was moderately associated with anxiety and depression. This is in line with earlier findings on the association between the ADNM-20 and the HADS in the initial validation of the ADNM-29 (Einsle et al., 2010) and could be another indicator of the applicability of the construct to the Chinese culture.

There are also several limitations to the present study. The generalizability of the results to the Chinese society is limited by the nature of the sample. University students from Hong Kong do not necessarily reflect the general Chinese population, thus replication with representative samples of Chinese adults across the socioeconomic and age spectrum is needed. The validation of a single assessment instrument of $\mathrm{AjD}$ does not allow final conclusions about the nature of the construct in a specific population. Research on $\mathrm{AjD}$ is still sparse and further studies should investigate the nature of $\mathrm{AjD}$ by using different means of assessment and in more representative samples.

\section{Conclusions}

Adjustment disorders are highly prevalent in primary care settings. However, there are only limited standardized assessments for adjustment disorder available. The Adjustment Disorder New Module 20 can facilitate the assessment of psychopathology as a result of critical life events. It is a self-report questionnaire that can be quickly employed and it can be evaluated according to the current ICD-11 guidelines for adjustment disorder. This can enable a quick assessment of the 
most relevant symptoms after life stress. The present study provides a significant contribution to the research of $\mathrm{AjD}$ as this was the first study to validate a structured assessment of $\mathrm{AjD}$ symptoms to the Chinese culture. The new conceptualization of $\mathrm{AjD}$ as stress-response syndrome was applicable among University students from Hong Kong and the Chinese version of ADNM-20 showed satisfactory properties regarding its content, factorial, and discriminant validity. More research is needed to assess its reliability in this cultural context. The translation and validation of a structured assessment for $\mathrm{AjD}$ can facilitate further research into cultural differences of stressrelated psychopathology.

\section{Declarations}

Acknowledgements: Not applicable.

Competing interests: The authors declare that they have no competing interests.

Funding details: This study was funded by the Start Up-Fund of The Hong Kong Polytechnic University [1-ZE7A]

Availability of data and material: The datasets used and/or analyzed during the current study are available from Grace W.K. Ho on reasonable request.

Biographical note: Louisa Lorenz is at the Department of Psychology, Division of Psychopathology and Clinical Intervention at University of Zurich. Her interest is in understanding and assessing adjustment and related disorders. Grace W.K. Ho is an Assistant Professor at the School of Nursing of The Hong Kong Polytechnic University. She is interested in understanding childhood adversities, resilience, and their impact on health and development. Athena C.Y. Chan is a Research Assistant at the School of Nursing of The Hong Kong Polytechnic University. She is interested in understanding the intergenerational transmission of parenting behaviors and their impact on children's psychosocial development. Daniel T. Bressington is an Assistant Professor at the School of Nursing of The Hong Kong Polytechnic 
University. He is interested in mental health promotion and health profiles of patients with severe mental illnesses. Wai Tong Chien is a Professor and Vice Director of Research at The Nethersole School of Nursing at The Chinese University of Hong Kong. He is interested in designing and testing interventions for community-resided people with mental health problems and their families. Mark Shevlin is a Professor of psychology at Ulster University. His research interests are in the areas of trauma, psychosis, and posttraumatic stress disorder. Philip Hyland is a Senior Lecturer at the Department of Psychology at Maynooth University. His primary research area is traumatic stress studies. Andreas Maercker is Professor and Head of Division for the Department of Psychology - Psychopathology and Clinical Intervention at University of Zurich. His expertise are in the areas of PTSD, stress-respond syndromes, lifespan developmental psychopathology, treatment, and e-mental health. Thanos Karatzias is Professor of Mental Health at Edinburgh Napier University and a Clinical \& Health Psychologist at the Rivers Centre for Traumatic Stress. His research focuses on the effects and treatment of psychological trauma on physical and mental health in vulnerable populations. 


\section{References}

American Psychiatric Association. (2013). Diagnostic and statistical manual of mental disorders (DSM-5®): American Psychiatric Pub.

Anderson, E. R., \& Mayes, L. C. (2010). Race/ethnicity and internalizing disorders in youth: A review. Clinical Psychology Review, 30(3), 338-348. doi:https://doi.org/10.1016/i.cpr.2009.12.008

Bachem, R., Perkonigg, A., Stein, D. J., \& Maercker, A. (2017). Measuring the ICD-11 adjustment disorder concept: Validity and sensitivity to change of the Adjustment Disorder New Module questionnaire in a clinical intervention study. International Journal of Methods in Psychiatric Research, 26(4), e1545. doi:10.1002/mpr.1545

Beaton, D. E., Bombardier, C., Guillemin, F., \& Ferraz, M. B. (2000). Guidelines for the process of cross-cultural adaptation of self-report measures. Spine, 25(24), 3186-3191.

Ben-Ezra, M., Mahat-Shamir, M., Lorenz, L., Lavenda, O., \& Maercker, A. (2018). Screening of adjustment disorder: Scale based on the ICD-11 and the Adjustment Disorder New Module. Journal of Psychiatric Research, 103, 91-96. doi:https://doi.org/10.1016/j.jpsychires.2018.05.011

Bujang, M. A., \& Baharum, N. (2017). A simplified guide to determination of sample size requirements for estimating the value of intraclass correlation coefficient: a review. Archives of Orofacial Science, 12(1).

Casey, P., Dowrick, C., \& Wilkinson, G. (2001). Adjustment disorders: Fault line in the psychiatric glossary. British Journal of Psychiatry, 179(6), 479-481.

doi:10.1192/bjp.179.6.479 
Chan, Y.-c., Lam, G. L. T., \& Shae, W.-C. (2011). Children's views on child abuse and neglect: Findings from an exploratory study with Chinese children in Hong Kong. Child Abuse \& Neglect, 35(3), 162-172. doi:https://doi.org/10.1016/j.chiabu.2010.12.001

Chen, F., Bollen, K. A., Paxton, P., Curran, P. J., \& Kirby, J. B. (2001). Improper Solutions in Structural Equation Models: Causes, Consequences, and Strategies. Sociological Methods \& Research, 29(4), 468-508. doi:10.1177/0049124101029004003

Chun, K. M., \& Hsu, J. (2012). Adjustment disorders in Asians. Handbook of Adult Psychopathology in Asians: Theory, Diagnosis, and Treatment. Oxford University Press, New York, NY, 328-356.

Colwell, S. R. (2016). The composite reliability calculator. Technical Report. doi:10.13140/RG.2.1.4298.088

DeVon, H. A., Block, M. E., Moyle-Wright, P., Ernst, D. M., Hayden, S. J., Lazzara, D. J., . . Kostas-Polston, E. (2007). A psychometric toolbox for testing validity and reliability. Journal of Nursing scholarship, 39(2), 155-164.

Einsle, F., Köllner, V., Dannemann, S., \& Maercker, A. (2010). Development and validation of a self-report for the assessment of adjustment disorders. Psychology, Health \& Medicine, 15(5), 584-595. doi:10.1080/13548506.2010.487107

Gau, S. S. F., Chong, M. Y., Chen, T. H. H., \& Cheng, A. T. A. (2005). A 3-Year Panel Study of Mental Disorders Among Adolescents in Taiwan. American Journal of Psychiatry, 162(7), 1344-1350. doi:10.1176/appi.ajp.162.7.1344

Glaesmer, H., Romppel, M., Brähler, E., Hinz, A., \& Maercker, A. (2015). Adjustment disorder as proposed for ICD-11: Dimensionality and symptom differentiation. Psychiatry Research, 229(3), 940-948. doi:https://doi.org/10.1016/i.psychres.2015.07.010 
Jackson, D. L., Gillaspy Jr, J. A., \& Purc-Stephenson, R. (2009). Reporting practices in confirmatory factor analysis: An overview and some recommendations. Psychological methods, 14(1), 6-23. doi:http://dx.doi.org/10.1037/a0014694

Jöreskog, K. G. (1994). On the estimation of polychoric correlations and their asymptotic covariance matrix. Psychometrika, 59(3), 381-389. doi:10.1007/BF02296131

Jöreskog, K. G., \& Sörbom, D. (1981). LISREL VI: Analysis of linear structural relationships by maximum likelihood, instrumental variables, and least squares methods: Scientific Software.

Jöreskog, K. G., \& Sörbom, D. (1993). LISREL 8: Structural equation modeling with the SIMPLIS command language: Scientific Software International.

Kazdin, A. E. (1977). Artifact, bias, and complexity of assessment: The ABCs of reliability. Journal of Applied Behavior Analysis, 10(1), 141-150.

Koo, T. K., \& Li, M. Y. (2016). A Guideline of Selecting and Reporting Intraclass Correlation Coefficients for Reliability Research. Journal of chiropractic medicine, 15(2), 155-163. doi:https://doi.org/10.1016/j.jcm.2016.02.012

Lavenda, O., Mahat-Shamir, M., Lorenz, L., Hamama-Raz, Y., Greenblatt-Kimron, L., PitchoPrelorentzos, S., . . B Ben-Ezra, M. (2019). Revalidation of Adjustment Disorder-New Module-4 screening of adjustment disorder in a non-clinical sample: Psychometric reevaluation and correlates with other ICD-11 mental disorders. Psych J, 8(3), 378-385. doi:10.1002/pchj.286

Li, Q., Lin, Y., Hu, C., Xu, Y., Zhou, H., Yang, L., \& Xu, Y. (2016). The Chinese version of hospital anxiety and depression scale: Psychometric properties in Chinese cancer patients and their family caregivers. European Journal of Oncology Nursing, 25, 16-23. doi:https://doi.org/10.1016/j.ejon.2016.09.004 
Lorenz, L., Bachem, R., \& Maercker, A. (2016). The adjustment disorder-new module 20 as a screening instrument: cluster analysis and cut-off values. The International Journal of Occupational and Environmental Medicine, 7(4 ), 215-220. doi:doi:

10.15171/ijoem.2016.775

Lorenz, L., Bachem, R. C., \& Maercker, A. (2016). The Adjustment Disorder--New Module 20 as a Screening Instrument: Cluster Analysis and Cut-off Values. Int J Occup Environ Med, $7(4), 215-220$.

Lorenz, L., Hyland, P., Perkonigg, A., \& Maercker, A. (2018). Is adjustment disorder unidimensional or multidimensional? Implications for ICD-11. International Journal of Methods in Psychiatric Research, 27(1), e1591. doi:10.1002/mpr.1591

Lynn, M. R. (1986). Determination and quantification of content validity. Nursing research, 35(6), 382-385. doi:10.1097/00006199-198611000-00017

Maercker, A., Brewin, C. R., Bryant, R. A., Cloitre, M., van Ommeren, M., Jones, L. M., .. . Reed, G. M. (2013). Diagnosis and classification of disorders specifically associated with stress: proposals for ICD-11. World Psychiatry, 12(3), 198-206. doi:10.1002/wps.20057

Maercker, A., Einsle, F., \& Köllner, V. (2007). Adjustment Disorders as Stress Response Syndromes: A New Diagnostic Concept and Its Exploration in a Medical Sample. Psychopathology, 40(3), 135-146. doi:10.1159/000099290

Muthén, B. O. (1997). Robust inference using weighted least squares and quadratic estimating equations in latent variable modeling with categorical and continuous outcomes. Psychometrika.

Muthén, L. K., \& Muthén, B. O. (2017). Mplus User's Guide (Eighth Edition ed.). Los Angeles, CA. 
Polit, D. F., \& Beck, C. T. (2006). The content validity index: are you sure you know what's being reported? Critique and recommendations. Research in nursing \& health, 29(5), 489497.

Polit, D. F., Beck, C. T., \& Owen, S. V. (2007). Is the CVI an acceptable indicator of content validity? Appraisal and recommendations. Research in nursing \& health, 30(4), 459-467.

Reise, S. P. (2012). The Rediscovery of Bifactor Measurement Models. Multivariate Behavioral Research, 47(5), 667-696. doi:10.1080/00273171.2012.715555

Shek, D. T. L., Chan, E. M. L., \& Wong, R. H. Y. (2012). Associations between Pathological Gambling and Psychiatric Comorbidity among Help-Seeking Populations in Hong Kong. The Scientific World Journal, 2012, 15. doi:10.1100/2012/571434

Strain, J. J., \& Diefenbacher, A. (2008). The adjustment disorders: the conundrums of the diagnoses. Comprehensive Psychiatry, 49(2), 121-130. doi:https://doi.org/10.1016/j.comppsych.2007.10.002

Svensson, E. (2012). Different ranking approaches defining association and agreement measures of paired ordinal data. Statistics in Medicine, 31(26), 3104-3117.

Tanaka, J. S. (1987). "How Big Is Big Enough?": Sample Size and Goodness of Fit in Structural Equation Models with Latent Variables. Child Development, 58(1), 134-146. doi:10.2307/1130296

The Hong Kong Polytechnic University. (2016). mySurvey (Version 1.1) [Software]. Retrieved from https://www.polyu.edu.hk/mysurvey/

Tu, C.-H., Hsu, M.-C., Chi, S.-C., Lin, H.-Y., \& Yen, Y.-C. (2014). Routine depression screening and diagnosing strategy for cancer inpatients. Psycho-Oncology, 23(9), 1057-1067. doi:10.1002/pon.3547 
Wang, W., Chair, S. Y., Thompson, D. R., \& Twinn, S. F. (2009). A psychometric evaluation of the Chinese version of the Hospital Anxiety and Depression Scale in patients with coronary heart disease. Journal of clinical nursing, 18(13), 1908-1915.

World Health Organisation. (2018). International Statistical Classification of Diseases and Related Health Problems, 11th revision (ICD-11). Retrieved from https://icd.who.int/browse11/1-m/en

World Health Organization. (1992). The ICD-10 classification of mental and behavioural disorders: clinical descriptions and diagnostic guidelines: Geneva: World Health Organization.

Zelviene, P., Kazlauskas, E., Eimontas, J., \& Maercker, A. (2017). Adjustment disorder: Empirical study of a new diagnostic concept for ICD-11 in the general population in Lithuania. European Psychiatry, 40, 20-25. doi:https://doi.org/10.1016/j.eurpsy.2016.06.009 Zigmond, A. S., \& Snaith, R. P. (1983). The hospital anxiety and depression scale. Acta Psychiatrica Scandinavica, 67(6), 361-370. 


\begin{tabular}{|c|c|c|c|c|c|c|c|}
\hline & \multirow[b]{2}{*}{ Item } & \multicolumn{3}{|c|}{ Relevance } & \multicolumn{3}{|c|}{ Appropriateness } \\
\hline & & $\mathrm{I}-\mathrm{CVI}$ & $\mathrm{P}_{\mathrm{c}}$ & $\mathrm{k}^{*}$ & $\mathrm{I}-\mathrm{CVI}$ & $\mathrm{P}_{\mathrm{c}}$ & $\mathrm{k}^{*}$ \\
\hline 1 & Since the stressful situation, I feel low and sad. & 1 & 0.016 & 1 & 1 & 0.016 & 1 \\
\hline 2 & I have to think about the stressful situation repeatedly. & 1 & 0.016 & 1 & 0.67 & 0.313 & 0.52 \\
\hline 3 & I try to avoid talking about the stressful situation whenever possible. & 1 & 0.016 & 1 & 1 & 0.016 & 1 \\
\hline 4 & I have to think about the stressful situation a lot and this is a great burden to me. & 1 & 0.016 & 1 & 0.67 & 0.313 & 0.52 \\
\hline 5 & I rarely do those activities, which I used to enjoy anymore. & 1 & 0.016 & 1 & 1 & 0.016 & 1 \\
\hline 6 & If I think about the stressful situation, I find myself in a real state of anxiety. & 1 & 0.016 & 1 & 0.83 & 0.094 & 0.82 \\
\hline 7 & I avoid certain things that might remind me of the stressful situation. & 1 & 0.016 & 1 & 1 & 0.016 & 1 \\
\hline 8 & I am nervous and restless since the stressful situation. & 1 & 0.016 & 1 & 1 & 0.016 & 1 \\
\hline 9 & Since the stressful situation, I lose my temper quicker than I used to, even over small things. & 1 & 0.016 & 1 & 1 & 0.016 & 1 \\
\hline 10 & Since the stressful situation, I find it difficult to concentrate on certain things. & 1 & 0.016 & 1 & 1 & 0.016 & 1 \\
\hline 11 & I try to dismiss the stressful situation from my memory. & 1 & 0.016 & 1 & 1 & 0.016 & 1 \\
\hline 12 & I have noticed that I am becoming more irritable due to the stressful situation. & 1 & 0.016 & 1 & 1 & 0.016 & 1 \\
\hline 13 & I constantly get memories of the stressful situation and can't do anything to stop them. & 1 & 0.016 & 1 & 1 & 0.016 & 1 \\
\hline 14 & I try to suppress my feelings because they are a burden to me. & 0.83 & 0.094 & 0.82 & 0.83 & 0.094 & 0.82 \\
\hline 15 & My thoughts often revolve around anything related to the stressful situation. & 1 & 0.016 & 1 & 1 & 0.016 & 1 \\
\hline 16 & $\begin{array}{l}\text { Since the stressful situation, I am scared of doing certain things or of getting into certain } \\
\text { situations. }\end{array}$ & 1 & 0.016 & 1 & 1 & 0.016 & 1 \\
\hline 17 & $\begin{array}{l}\text { Since the stressful situation, I do not like going to work or carrying out the necessary tasks of } \\
\text { everyday life. }\end{array}$ & 1 & 0.016 & 1 & 1 & 0.016 & 1 \\
\hline 18 & I have been feeling dispirited since the stressful situation and have little hope for the future. & 1 & 0.016 & 1 & 1 & 0.016 & 1 \\
\hline 19 & Since the stressful situation, I can no longer sleep properly. & 1 & 0.016 & 1 & 1 & 0.016 & 1 \\
\hline 20 & $\begin{array}{l}\text { All in all, the situation causes serious impairment in my social or occupational life, my } \\
\text { leisure time, and other important areas of functioning. }\end{array}$ & 1 & 0.016 & 1 & 1 & 0.016 & 1 \\
\hline
\end{tabular}

Note. $\mathrm{I}-\mathrm{CVI}=$ item content validity index. $\mathrm{P}_{\mathrm{c}}=$ probability of a chance occurrence. $\mathrm{k}^{*}=$ kappa designating agreement on relevance. All kappa values can be evaluated as excellent, except for appropriateness of items 2 and 4 
Table 2. Fit indices for alternative models of the structure of adjustment disorder $(N=433)$

\begin{tabular}{|c|c|c|c|c|c|c|}
\hline Model & $\chi^{2}$ & $\mathrm{df}$ & CFI & TLI & RMSEA $(95 \% \mathrm{CI})$ & SRMR \\
\hline 1-factor with correlated error between items 9 and 12 & 541.702 & $151^{*}$ & .964 & .959 & $.077(.070 ; .084)$ & .045 \\
\hline 5-factor with correlated error between items 9 and 12 & 395.715 & $141^{*}$ & .977 & .972 & $.065(.057 ; .072)$ & .037 \\
\hline 6-factor (Einsle et al., 2010) & 475.456 & $137 *$ & .969 & .961 & $.076(.068 ; .083)$ & .039 \\
\hline 6-factor with correlated error between items 9 and 12 & 382.271 & $136^{*}$ & .977 & .972 & $.065(.057 ; .072)$ & .036 \\
\hline \multicolumn{7}{|l|}{ Core symptoms } \\
\hline 2-core-factor (Zelviene et al., 2017) & 49.227 & 13 & .988 & .981 & $.080(.057 ; .105)$ & .024 \\
\hline
\end{tabular}

Note. CFI=Comparative Fit Index; TLI=Tucker-Lewis-Index; RMSEA=Root-Mean Square Error of Approximation; SRMR=Standardized Root Mean Square Residual; SRMR $=$ Standardized Root Mean Square Residual

$* \mathrm{p}<.05$ 
Table 3. Percentage agreement, systematic disagreement and individual variability of ADNM20 items at test and retest $(n=32)$

\begin{tabular}{|c|c|c|c|c|}
\hline Item & $\mathrm{PA}$ & $\mathrm{RP}(95 \% \mathrm{CI})$ & $\mathrm{RC}(95 \% \mathrm{CI})$ & RV (95\% CI) \\
\hline 1 & 53 & $-0.26 *(-0.444 ;-0.065)$ & $-0.06(-0.33 ; 0.21)$ & $0.05(0 ; 0.134)$ \\
\hline 2 & 44 & $-0.15(-0.392 ; 0.099)$ & $-0.18(-0.36 ; 0)$ & $0.39(0.006 ; 0.773)$ \\
\hline 3 & 56 & $-0.04(-0.206 ; 0.120)$ & $0.16(-0.01 ; 0.32)$ & $0.07(0 ; 0.171)$ \\
\hline 4 & 53 & $-0.01(-0.238 ; 0.216)$ & $0.01(-0.16 ; 0.18)$ & $0.24(0.017 ; 0.464)$ \\
\hline 5 & 47 & $-0.08(-0.261 ; 0.105)$ & $-0.01(-0.26 ; 0.24)$ & $0.12(0 ; 0234)$ \\
\hline 6 & 44 & $0.05(-0.106 ; 0.206)$ & $0.06(-0.20 ; 0.31)$ & $0.04(0 ; 0.081)$ \\
\hline 7 & 63 & $>-0.01(-0.152 ; 0.140)$ & $-0.01(-0.19 ; 0.17)$ & $0.08(0 ; 0.190)$ \\
\hline 8 & 38 & $-0.08(-0.269 ; 0.101)$ & $0.10 *(0.15 ; 0.35)$ & $0.09(0.015 ; 0.164)$ \\
\hline 9 & 47 & $-0.07(-0.245 ; 0.100)$ & $0.20(-0.01 ; 0.41)$ & $0.08(0 ; 0.177)$ \\
\hline 10 & 44 & $-0.13 *(-0.316 ;-0.006)$ & $0.27(0.05 ; 0.49)$ & $0.03(0 ; 0.057)$ \\
\hline 11 & 59 & $-0.12(-0.329 ; 0.087)$ & $0.18 *(0.01 ; 0.34)$ & $0.16(0 ; 0.319)$ \\
\hline 12 & 44 & $-0.27 *(-0.430 ;-0.115)$ & $-0.13(-0.39 ; 0.12)$ & $0.05(0 ; 0.106)$ \\
\hline 13 & 59 & $-0.07(-0.235 ; 0.096)$ & $0.01(0.18 ; 0.21)$ & $0.06(0 ; 0.145)$ \\
\hline 14 & 56 & $-0.12(-0.315 ; 0.069)$ & $0.11(-0.11 ; 0.32)$ & $0.17(0 ; 0.357)$ \\
\hline 15 & 44 & $-0.18(-0.365 ; 0.014)$ & $0.17(-0.07 ; 0.42)$ & $0.13(0.004 ; 0.264)$ \\
\hline 16 & 50 & $-0.12(-0.311 ; 0.077)$ & $0.05(-0.15 ; 0.25)$ & $0.15(0 ; 0.307)$ \\
\hline 17 & 56 & $<0.01(-0.142 ; 0.146)$ & $0.15(-0.08 ; 0.37)$ & $0.03(0 ; 0.067)$ \\
\hline 18 & 56 & $-0.17 *(-0.318 ;-0.012)$ & $-0.03(-0.26 ; 0.20)$ & $0.06(0 ; 0.149)$ \\
\hline 19 & 53 & $-0.06(-0.246 ; 0.137)$ & $-0.03(-0.23 ; 0,17)$ & $0.15(0 ; 0.304)$ \\
\hline 20 & 56 & $-0.14(-0.306 ; 0.035)$ & $0.19(0 ; 0.39)$ & $0.05(0 ; 0.122)$ \\
\hline
\end{tabular}

Note. $\mathrm{PA}=$ percentage agreement; $\mathrm{RP}=$ relative position; $\mathrm{RC}=$ relative concentration; $\mathrm{RV}=$ relative rank variance; $95 \% \mathrm{CI}=95 \%$ confidence interval; $\mathrm{RP} / \mathrm{RC} \geq|0.1|$ are in bold.

* Statistically significant non-negligible disagreement $(\mathrm{RP} / \mathrm{RC} \geq|0.1|$ and $95 \% \mathrm{CI}$ that do not cover zero) 